\title{
FLUXO DE ENERGIA EM COMUNIDADES AQUÁTICAS, COM ÊNFASE EM ECOSSISTEMAS LÓTICOS
}

\author{
Carla Ferreira Rezende ${ }^{1,2,3^{*}}$, Érica Maria Pellegrini Caramaschi ${ }^{1}$ \& Rosana Mazzoni ${ }^{2}$ \\ ${ }^{1}$ Laboratório de Ecologia de Peixes, Instituto de Biologia, Departamento de Ecologia, Universidade Federal do Rio de Janeiro, Av. Mal. Trompowski, \\ s/n CCS Bloco A - Ilha do Fundão, CEP 21941-590 - Rio de Janeiro, Brasil \\ ${ }^{2}$ Laboratório de Ecologia de Peixes, Departamento de Ecologia, Universidade do Federal do Rio de Janeiro, Av. Mal. Trompowski, s/n CCS Bloco \\ A - Ilha do Fundão, CEP 21941-590 - Rio de Janeiro, Brasil \\ ${ }^{3}$ Laboratório de Ecologia de Peixes, Instituto de Biologia Roberto Alcantara Gomes, Departamento de Ecologia, Universidade Estadual do Rio de \\ Janeiro, Rua São Francisco Xavier 524, Maracanã, CEP 20550013 - Rio de Janeiro, Brasil \\ *E-mail: carla.fr@ufrj.br
}

\section{RESUMO}

Os trabalhos de Lindeman (1942), seguidos pelos trabalhos de Odum $(1956,1957)$, desperteam o interesse de ecólogos sobre questões a cerca da produção biótica e do fluxo de energia em sistemas aquáticos. A ecologia energética ocupa-se fundamentalmente da forma como a energia transita no interior do sistema. Ao nível ecossistêmico o fluxo de energia desenvolve uma série de reações químicas que podem liberar ou absorver calor. Essas funções atuam primeiramente ao nível individual, populacional (Odum \& Smalley 1959) e, posteriormente, ecossistêmico (Odum 2001). Odum foi o primeiro a desenvolver uma abordagem para medir o estado de maturidade do sistema que era atingido quando observado incremento de biomassa. Essa avaliação termodinâmica media o estágio de maturidade dos ecossistemas, e verificava o desempenho de cada componente, alicerçado ao manejo de populações naturais. A aplicação da teoria de Odum pode ser desenvolvida com análises sobre redes tróficas que permitem quantificar o estágio de evolução de um ecossistema, através das propriedades emergentes. A ausência de dados quantitativos (biomassa) englobando todos os organismos do ecossistema junto com a dificuldade de se encontrar ferramentas para o desenvolvimento desses trabalhos tem sido uma das principais dificuldades dessas abordagens. A presente revisão tem como objetivo traçar um histórico da ecologia energética em sistemas aquáticos, enfatizando as principais metodologias utilizadas para verificar o fluxo de energia nos sistemas ecológicos.

Palavras-chave: Ecologia energética, rios, peixes, teias tróficas

\begin{abstract}
ENERGY FLOW IN AQUATIC COMMUNITIES, EMPHASIZING LOTIC SYSTEMS. A series of studies by Lindeman and Odum attracted scientific attention to the matter of biotic production and energy flow in aquatic ecosystems. Energy flow ecology fundamentally deals with how energy is transferred in a given system. The flow of energy generates a series of chemical reactions which may absorb or release heat, and these heat variations occur at the level of the individual, with subsequent effects over the entire population and ecosystem. Odum was the first author to develop a thermodynamic method to measure the state of maturity reached by a given system following biomass increment. This method was used to evaluate the individual performance of each of the ecosystem's parts based on the natural processes intrinsic to populations. Odum's theory can be further developed by including trophic web analysis, thus allowing assessment of the evolutionary stage of ecosystems based on emergent properties. The greatest obstacles for such an approach is the lack of quantitative data (biomass) that would include all organisms of any given ecosystem and the difficulty in finding useful tools to develop these studies. The objective of the present review is to present an historical account of the energy flow ecology in aquatic ecosystems, focusing on the main methodologies employed.
\end{abstract} Keywords: Energy flow, rivers, fishes, isotopes, trophic webs. 


\section{INTRODUÇÃO}

O interesse sobre a produção biótica e o fluxo de energia em sistemas aquáticos foi impulsionado, inicialmente pelos trabalhos de Lindeman (1942) que abordavam a dinâmica trófica de comunidades e pelos trabalhos de Odum $(1956,1957)$ que aprimoraram o estudo do fluxo de energia em sistemas. Nesse período, os pesquisadores da época foram atraídos pelo tema e a compreensão sobre dinâmica e funcionamento de sistemas lóticos se desenvolveu. Esse período, entre meados de 1950 e início da década de 1980, ficou conhecido como Era da Produção Biótica e do Fluxo de Energia (Minshall 1988).

A ecologia energética ocupa-se fundamentalmente da forma como a energia transita no interior do sistema. Sendo, as relações entre produtores e consumidores, predador e presa, limitadas e regidas pelas mesmas leis básicas dos sistemas vivos e controladas pelo fluxo de energia (Odum 1988). Ao nível ecossistêmico o fluxo de energia desenvolve uma série de reações químicas que podem liberar ou absorver calor (Odum 2001). Essas funções atuam primeiramente ao nível individual, populacional (Odum \& Smalley 1959) e posteriormente ecossistêmico (Odum 2001). Sendo relacionadas com a desordem interna (entropia), com o conteúdo de calor (entalpia), com a energia livre e com o armazenamento de energia dos organismos (Scott 1965).

Para o termo ecossistema, Odum (1956) descreveu 24 atributos ligados ao fluxo interno, controle de retroalimentação e crescimento da diversidade. Dentro desses atributos, os cinco primeiros representaram a bioenergética do ecossistema (Odum 2001). Nessa abordagem, o estado de maturidade do sistema era atingido quando observado incremento de biomassa, especialmente de animais de grande porte com altas taxas de longevidade e aumento na ciclagem dos detritos através do aumento da complexidade da teia (Pauly et al. 2000). Essa avaliação termodinâmica media o estágio de maturidade dos ecossistemas, e verificava o desempenho de cada componente, alicerçado ao manejo de populações naturais (Angelini 2002).

A aplicação da teoria de Odum pode ser desenvolvida com análises sobre redes tróficas que permitem quantificar o estágio de evolução de um ecossistema, através das propriedades emergentes.
Por esta razão, houve uma discussão sobre teias tróficas como sinônimos de ecossistema (Angelini \& Agostinho 2005). As propriedades emergentes poderiam ser consideradas medidas de avaliação dos estados e amadurecimento do sistema, já que estas só "emergiriam" quando as partes do sistema estivessem interligadas (Christensen 1995, Angelini 2002). Para se avaliar o ecossistema tomou-se importante conhecer o sistema como um todo, considerando todas as suas partes, ligações e principalmente as propriedades emergentes. Intuitivamente isto foi proposto por Eugene P. Odum (1969) com o conceito de maturidade, sugerindo que o ecossistema apresentava um desenvolvimento sucessional que, em determinado momento, atingiria a maturidade. Esses estados sucessionais apresentavam uma ordem cronológica e direcional que poderia ser prevista. $\mathrm{O}$ desenvolvimento atingia seu ponto máximo com a estabilidade do sistema e com a quantidade máxima de biomassa e/ou informação. Recentemente, a teoria do caos tem demonstrado que não é tão simples predizer estes estados de desenvolvimento, mas as descrições de Odum ainda são generalizações que nos direcionam a verificar o desenvolvimento do sistema (Christensen 1995). Apesar da influência e de algumas críticas às generalizações de Odum quanto à maturidade do ecossistema, poucas interpretações quantitativas emergiram nas últimas duas décadas desde suas propostas.

A ausência de dados quantitativos (biomassa) englobando todos os organismos do ecossistema junto com a dificuldade de se encontrar ferramentas para o desenvolvimento desses trabalhos tem sido uma das principais dificuldades dessas abordagens. Houve uma tentativa do "International Biological Programme" (IBP) de realizar modelagem com integração em níveis tróficos, utilizando base de dados de biomassa e produção de cada nível trófico. Estes dados eram enviados para "modeladores" que desenvolviam simulação com esses bancos (Pauly et al. 2000). A principal crítica a esses modelos foi o agrupamento em níveis tróficos. Rigler (1975) criticou o agrupamento, argumentando que a maioria dos organismos aquáticos se alimentava simultaneamente em mais de um nível trófico. A solução para este problema foi sugerida por Odum \& Heald (1975) através de uma modelagem de fracionamento de níveis tróficos. Atualmente, este 
fracionamento pode ser calculado com base nos isótopos estáveis de nitrogênio ou com alguns modelos que conseguem modelar a onívora através de um índice baseado na composição da dieta e que apresentam resultado equivalente ao encontrado pelas estimativas através dos isótopos (Kline \& Pauly 1998).

Neste contexto, a presente revisão tem como objetivo apresentar o histórico da ecologia energética em sistemas aquáticos, enfatizando as principais metodologias utilizadas na investigação do fluxo de energia nos sistemas ecológicos.

\section{ENERGIA}

A definição de energia surgiu quando um engenheiro francês, N.L. Sadi Carnot publicou os resultados parciais de uma pesquisa com o título: "Reflexões sobre potência motriz do fogo", onde estudando o desempenho de maquinas térmicas, em que descreveria a equivalência constante entre calor absorvido e calor cedido pela máquina. Pouco tempo depois J.P. Joule comunicou seus resultados sobre equivalência térmica do trabalho ou equivalência térmica do calor, afirmando que quando a quantidade de "força viva" é aparentemente destruída, é produzida uma quantidade equivalente de calor, e a relação permanece válida para o processo inverso, denominada de Equivalência Térmica do Trabalho ou Equivalência Mecânica do Calor (Baptista 2006).

Posteriormente, a força viva foi batizada com o nome de energia e a lei de equivalência confirmou a interpretação de Joule do calor como uma fonte e a energia térmica, como uma energia de trânsito (Baptista 2006). Atualmente, a energia é definida como a capacidade de realizar trabalho e esta definição é extremamente importante para os sistemas vivos, já que o desempenho interno do trabalho é necessário para manutenção do organismo vivo (Scott 1965, Wiegert 1968). Todos os organismos realizam trabalho para viver, tendo a energia um significado de continuidade e expansão da vida para o organismo (Wiegert 1968). Por esta razão, a energia e as funções termodinâmicas dos organismos e dos sistemas vivos são temas muito abordados em trabalhos de fisiologia e ecologia (Scott 1965).

Há várias formas reconhecidas de energia, mas as de maior importância para os organismos vivos são: mecânica, química, radiante e calorífica. A energia mecânica tem duas formas: cinética e potencial. Energia cinética é medida pela quantidade de trabalho que é executado para trazer o corpo ao repouso. Energia potencial é a energia reservada, apenas potencialmente utilizável, até sua conversão na cinética, quando se torna viável para produzir trabalho. A energia química é a fonte potencial de energia encontrada no alimento, a qual, é liberada através do rearranjo do conjunto de átomos na matéria, que proporcionam energia mecânica. Já a energia radiante é a energia na forma de ondas eletromagnéticas, envolvendo troca entre energia potencial e energia cinética. Por último, a energia calorífica resulta do movimento ao acaso de moléculas. Para os animais, a fonte básica de energia são os vegetais, que agem como uma reserva de energia potencial, que é convertida em cinética quando é necessária para realizar trabalho. Essas formas de energia mecânica (potencial e cinética) são geradas através das reações combustão (oxidação) do alimento (Phillipson 1969).

Neste sendito, a primeira lei da termodinâmica afirma que a energia pode transformar-se em trabalho, calor ou energia potencial de alimentos, conforme a situação, embora nenhuma porção dela possa ser destruída. A segunda lei da termodinâmica pode ser enunciada de diversos modos, incluindo o seguinte: nenhum processo envolvendo uma transformação de energia ocorrerá espontaneamente a menos que haja uma degradação de energia de uma forma concentrada para uma forma dispersa (Odum 2001).

\section{HISTÓRICO E APLICAÇÃO DA ENERGIA EM SISTEMAS BIOLÓGICOS}

Charles Elton (1900-1991) foi o primeiro ecólogo a se interessar pela estrutura da comunidade biológica e descrever sua dinâmica. Este pesquisador descreveu a estrutura das cadeias alimentares, pirâmides de números e surgiu com os primeiros estudos sobre teias tróficas e fluxo de energia entre os organismos de uma comunidade biológica, desenvolvendo um importante conceito na ecologia "nicho ecológico" (Kingsland 1991).

Raymond Lindeman (1915-1942), um ecólogo que teve suas idéias impulsionadas pelos trabalhos de Elton, desenvolveu as idéias que emergiram no paradigma da dinâmica trófica e estudos sobre fluxo de energia em cadeias alimentares. Essas idéias que tornaram-se 
freqüentes em na Ecologia e surgiram a partir de sua tese de doutorado. A síntese dessa pesquisa foi publica em 1942, intitulada por Lindemann como "trophicdynamic aspects". Através de seus estudos de fisiologia e ecologia de comunidades, esse pesquisador com base em relações nutricionais e tróficas conseguiu descrever importantes padrões para comunidades em sucessão (Kingsland 1991). Outro ecólogo que influenciou os conceitos desenvolvidos por Lindemann foi G. Evelyn Hutchinson que juntamente com A. Wollack em 1940 desenvolveu um trabalho sobre transferência de energia, processos tróficos e sucessão.

O conceito de ecossistema também foi abordado por Lindemann. Esse conceito foi introduzido com pouco impacto na ecologia por Though Arthur Tansley em 1935, no entanto após a inclusão do contexto de dinâmica sucessional, foi fortalecido e pode ser mais aplicado e utilizado em trabalhos de sucessão (Kingsland 1991).

Porém a difusão do conceito de ecossistema e os avanços nos trabalhos envolvendo princípios termodinâmicos foram impulsionados pelos irmãos Howard Odum (1924-2002) e Eugene Odum (19132002), após a publicação do livro 'Fundamentos de Ecologia'. Nesse período alguns pesquisadores concentraram suas pesquisas em abordagens ecossistêmicas, com ênfase na termodinâmica e na teoria da informação para compreender o fluxo de energia no ecossistema (Odum \& Pinkerton 1955, Patten 1959, 1961, Slobodkin 1960, Wiegeret 1964).

O ponto de partida para a aplicação dos modelos termodinâmicos foi a padronização da unidade energética para determinar as relações do fluxo de energia. Assim, primeiramente, foi discutida a utilização de medidas de biomassa, já que, na química utiliza-se unidade mol. $\mathrm{Na}$ abordagem biológica essa unidade não é utilizada, pois os organismos vivos são compostos por uma mistura de componentes, sendo impossível a distinção em unidades mol (Scott 1965). No século XVIII, foi verificado que a energia potencial poderia ser medida pela simples multiplicação: peso levantado $\mathrm{X}$ altura erguida. No mesmo período o calor foi medido com exatidão através da relação: massa de água $\mathrm{X}$ elevação da temperatura, sendo expressa pela unidade caloria (cal) ou quilocaloria (Kcal) (Phillipson 1969). Uma vez que todas as formas de energia podem ser convertidas em calor, a caloria tornou-se uma unidade muito utilizada para trabalhos comparativos, prin- cipalmente de ecologia energética (Phillipson 1969, Dourado \& Benedito-Cecíllio 2005).

A quantificação de energia em unidades de calor pode ser medida através de uma bomba calorimétrica (Scott 1965, Phillipson 1969), onde o peso conhecido de uma substância sofre combustão completa em um recipiente de metal isolado e, medindo-se a quantidade de calor produzido no calorímetro de bomba, o valor calorífico da substância é calculado (Scott 1965, Phillipson 1969, Benedito-Cecíllio 2005). Outra forma de calcular a energia de um dado organismo é através da conversão da biomassa (peso) em fator energético ou energia equivalente (Scott 1965). Se a composição química do organismo é conhecida, essa equivalência pode ser obtida a partir de padronizações provenientes de dados de trabalhos de termodinâmica. Este tipo de conversão tem sido amplamente difundido (Christensen \& Pauly 1992). No entanto, sabe-se que organismos vivos apresentam uma composição química complexa e que podem modificar rapidamente em respostas a variáveis ambientais (Scott 1965, Doria \& Adrian, 1997). Por isso, medidas diretas através de calorímetros são mais precisas do que medidas de conversão (Scott 1965).

Asmedidas debiomassa, aplicadas em propriedades termodinâmicas, foram utilizadas para a verificação do fluxo de energia nos sistemas (Polovina 1984, Christensen \& Pauly 1992). Importantes conceitos foram desenvolvidos desde os primeiros trabalhos de Lindeman (1942) e Odum (1969). Trabalhos recentes (e.g. Wilson \& Parkes 1998, Meyer \& Poepperl 2004, Angelini \& Agostinho 2005) associados a equações de balanceamento de massa ainda seguem esta abordagem, considerando aspectos relacionados à visão ecossistêmica e sendo aplicados em projetos de manejo e conservação (Christensen et al. 2005). No entanto, com o desenvolvimento de técnicas de isótopos estáveis, surgiu uma nova abordagem na determinação dos níveis tróficos dentro das comunidades (Vander-Zanden \& Rasmussen 2001), inovando os trabalhos de fluxo de energia. Essa técnica vem auxiliando a delinear e melhor compreender a dinâmica trófica entre os organismos produtores, consumidores e decompositores nas teias alimentares. Com base na composição isotópica dos tecidos dos consumidores, tem-se a indicação mais precisa do item assimilado dentre os alimentos consumidos (Manetta \& Benedito-Cecilio 2003). 


\section{ISÓTOPOS ESTÁVEIS}

O termo isótopo, em 1913 por Frederick Soddy (1877-1956), é definido como sendo átomos do mesmo elemento com os mesmos números de prótons, mas com diferentes números de nêutrons, ou seja, com diferentes números de massa. Já o principio do primeiro espectrômetro de massa foi visualizado por Francis William Aston (1877-1945) que estabeleceu evidências de que o conceito de isótopo aplicava-se a todos os elementos e não apenas aos radioativos.

Os elementos ocorrem naturalmente em várias formas isotópicas, sendo que algumas são instáveis e por isso sua radioatividade decai com o tempo. Outros isótopos não apresentam diminuição radioativa sendo considerados isótopos estáveis. Estes isótopos são utilizados a partir de padronizações calculadas com valores das amostras e de referência do ambiente natural. $\mathrm{O}$ isótopo pode apresentar enriquecimento ou depleção, apresentando-se na forma pesada ou leve. Essas formas têm pequenas diferenças em propriedades termodinâmicas e em relação à quantidade de massa. Essas diferenças termodinâmicas podem levar a distintas taxas de reações bioquímicas e conseqüentemente a diferentes taxas cinéticas de isótopos pesados e leves em reações fisiológicas resultando em fracionamento, ou mudança na taxa de isótopos encontrados em um organismo relativo ao seu recurso utilizado (Manetta \& Benedito-Cecílio 2003). Os isótopos estáveis mais utilizados para este objetivo são carbono $\left(\delta^{13} \mathrm{C}\right)$ e nitrogênio $\left(\delta^{15} \mathrm{~N}\right)$. Os resultados baseiam-se na determinação da proporção

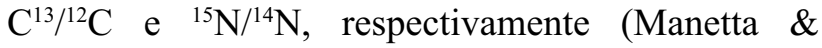
Benedito-Cecílio 2003).

Os isótopos estáveis $\delta^{13} \mathrm{C}$ e $\delta^{15} \mathrm{~N}$ são utilizados em trabalhos de teias tróficas e fluxo de energia (VandenZanden \& Rasmussen 2001). A composição isotópica de carbono nos tecidos animais apresenta um enriquecimento por nível trófico de aproximadamente $1 \%$, evidenciado pelo valor isotópico do carbono no animal em relação ao valor isotópico do carbono na dieta. Além disso, a transferência da assinatura isotópica de carbono ao longo da teia trófica é conservativa podendo ser utilizada para traçar o fluxo de energia em sistemas onde existem vários tipos de alimentos com diferenças nos valores de ${ }^{13} \mathrm{C}$ (Manetta \& Benedito-Cecílio 2003). Já o isótopo estável do nitrogênio $\left(\delta^{15} \mathrm{~N}\right)$ é utilizado para determinar o nível trófico a que uma determinada espécie pertence. Esse isótopo tem um padrão de fracionamento em que ocorre enriquecimento de 3\%o à medida em que se aumenta o nível trófico (DeNiro \& Epstein 1981, Minigawa \& Wada 1984, Manetta \& Benedito-Cecílio 2003). Desta forma, o isótopo do carbono é utilizado para distinguir as fontes de energia terrestre, aquática ou marinha. Enquanto o isótopo do nitrogênio é utilizado para a determinação do nível trófico na teia alimentar.

Após 30 anos da aplicação de isótopos estáveis como ferramenta em trabalhos de ecologia, já há um consenso da comunidade científica sobre a utilidade de sua aplicação como bons traçadores naturais do fluxo de energia em teias tróficas de ecossistemas aquáticos (Michener \& Shell 1994). Os primeiros trabalhos relacionando a composição isotópica de carbono em animais com a dieta foram desenvolvidos por Haines (1976), DeNiro \& Epstein (1978), Fry et al. (1978), Haines \& Montague (1979), Teeri \& Schoeller (1979), Rau (1980), Rau \& Andersen (1981), Fry \& Arnold (1982), Tieszen et al. (1983), Checkley \& Entzeroth (1985), Peterson \& Fry (1987) que verificaram que o enriquecimento da dieta em carbono era refletido em 1\%. Atualmente, sabe-se que este pode variar de 0.5-1\%o (Michener \& Shell 1994).

Contrastando com o carbono, inicialmente menos trabalhos foram destinados a verificar relação entre o nitrogênio, os organismos e sua dieta (Gaebler et al. 1966, Steele \& Daniel 1978, Rau 1981, DeNiro \& Epstein 1981, Checkley \& Entzeroth 1985, Peterson \& Fry 1987). No entanto, eles foram de suma importância para comprovar o enriquecimento deste isótopo de acordo com o aumento do nível trófico.

$\mathrm{Na}$ América do Sul, as pesquisas realizadas com análises de isótopos têm criado controvérsias, ao indicar que as microalgas são as fontes predominantes de energia para muitos organismos em riachos (Moulton 2006). Hamilton et al (1992) observaram esse padrão para os invertebrados e peixes da várzea do rio Orinoco. Na bacia do Paraná também foi verificado que as plantas $\mathrm{C}_{4}$ responsáveis por grande parte do detrito disponível na planície de inundação, não apresentaram grande importância na dieta de peixes detritívoros (Vaz et al. 1999). Enquanto Lopes et al. (2007) encontraram variabilidade quanto ao carbono utilizado por Prochilodus lineatus em diferentes ambientes do mesmo ecossistema, Benedito-Cecilio et al. (2004) registraram para espécies da Amazônia 
predominância de $\mathrm{C}_{3}$, podendo ser proveniente das algas ou da vegetação ripária. Pereira et al. (2007), por outro lado, apontam variações na abundância de $\mathrm{C}_{4}$ e $\mathrm{C}_{3}$ na dieta de Leporinus friderici, assim como Manetta et al. (2003), que também verificaram predominância de $\mathrm{C}_{3}$ na dieta dos peixes do Rio Baía, planície de inundação do alto rio Paraná..

Em um lago do Pantanal, mudanças isotópicas, de acordo com o gradiente sazonal, foram verificadas para espécies de peixes, como Brycon microlepsis, que apresentou variações de 13 até $30 \%$ do carbono de plantas $\mathrm{C}_{4}$ durante a cheia (Wantzen et al. 2002). $\mathrm{Na}$ região amazônica, plantas $\mathrm{C}_{4}$ tiveram grande contribuição para a dieta das larvas de peixe, enquanto as $\mathrm{C}_{3}$ foram predominantes na dieta dos adultos (Leite et al. 2002). Benedito-Cecilio et al. (2000), por sua vez, verificaram variações na contribuição das fontes em função do pulso de inundação. No caso das espécies de peixes detritívoras, foi surpreendente o fato de fitoplâncton e COP serem suas principais fontes de energia (Araújo-Lima et al. 1986). Posteriormente, outros trabalhos da região amazônica demonstraram o mesmo padrão da importância do carbono $\mathrm{C}_{3}$ para o sistema (Benedito-Cecilio \& Araújo-Lima. 2002).

\section{PARADOXO SOBRE A ORIGEM DA ENERGIA NOS SISTEMAS LÓTICOS}

Os ecossistemas são dependentes de duas fontes de energia: a radiação solar fixada, através da fotossíntese e da matéria orgânica proveniente de fontes alóctones (Fisher \& Likens 1973, Vannote et al 1980). Essas diferentes fontes de energia atuam de forma que, ao longo do gradiente, as taxas de produção/respiração se intercalem, sendo os trechos superior e inferior heterotróficos, e o trecho médio, autotrófico (Vannote et al. 1980). O conceito do Rio Contínuo proposto por Vannote et al. (1980) está sendo contestado pelo modelo (River Productivity Model), modelo de produtividades de rios, que implica na entrada de energia nos rios de quarta ordem, através das algas. Esse modelo discute o seguinte paradoxo: Como pode a biomassa animal dentro da teia trófica dos rios ser abastecida pela produção primária autotrófica se o ecossistema como um todo é heterotrófico? (Thorp \& Delong 1994, 2002).

A utilização de isótopos estáveis para medir fluxo de energia trouxe esse paradoxo para a ecologia de riachos, pois, embora a entrada de biomassa alóctone no sistema seja grande (e.g. Minshall 1967, Cummins 1974, Vannote et al 1980, Rounick \& Winterbourn 1986, Wallace et al. 1997, Moulton 2006), o carbono proveniente da serrapilheira é menos assimilado do que o carbono proveniente das algas (Thorp \& Delong 2002, Moulton 2006), devido a uma diferenciação na taxa de assimilação entre os dois tipos de carbono (Gaedke et al 1996).

Os primeiros trabalhos relacionando a origem do carbono (autóctone ou alóctone) em riachos foram desenvolvidos por Rounick et al. (1982), Winterbourn et al. (1984) e Rounick \& Winterbourn (1986) na Nova Zelândia. Esses autores verificaram que, para o riacho florestado, o aporte de carbono alóctone era mais representativo, enquantono riachodesmatadoo aporte de carbono autóctone era mais importante. Nesse trabalho, os autores comentaram que as análises com isótopos de carbono poderiam não apenas indicar as fontes de alimentação dos organismos, mas novos caminhos de ciclagem de carbono associados à microflora. Pois baixa depleção de $\delta^{13} \mathrm{C}$ encontrada nos invertebrados poderia ser explicada pela presença de microorganismos que se utilizariam desta via. Posteriormente, Rosenfeld \& Roff (1992) corroboraram esta relação em um riacho do Canadá, mas recomendaram complementações de análises de conteúdo estomacal e análises com carbono estável ao longo do ano para avaliar a consistência do padrão encontrado.

Moulton (2006), ao tratar da predominância das algas na assinatura isotópica em teias tróficas de riachos citou uma série de trabalhos que corroboram o modelo RPM (River Productivity Model). Corroborando estes trabalhos Brito et al. (2006) em um córrego da Ilha Grande (RJ), demonstrou que apesar da biomassa alóctone ser maior do que a de perifiton (algas), a teia trófica do riacho foi baseada em microalgas ao contrário do evidenciado por análises de conteúdo estomacal das espécies deste mesmo riacho (Rezende \& Mazzoni 2005, Moulton 2006). Segundo Brito et al. (2006) uma hipótese para explicar este padrão seria a de que nos riachos tropicais a produção das algas seria mantida mesmo em ambientes sombreados, contrastando com as regiões temperadas (Mosisch et al. 2001, Finlay 2001).

Em variados tipos de sistema na região neotropical estudos vêm demonstrando grande importância das microalgas como fonte de energia para o sistema aquático (e.g. Araújo-Lima 1986, Hamilton et al.1992, Vaz et al. 1999, Wantzen et al. 2002, Forsberg et al. 
1993, Benedito-Cecilio et al. 2000, Waichman 1996, Salas \& Dudgeon 2001, Leite et al. 2002, BeneditoCecilio \& Araújo-Lima 2002, March \& Pringle 2003, Benedito-Cecilio et al. 2004, Mantel et al. 2004, Brito et al.2006). No entanto, os estudos nesta área ainda são insuficientes, sendo necessários mais estudos de caso, tanto descritivos como experimentais, envolvendo análises de isótopos para corroborar o modelo proposto por Thorp \& Delong $(1994,2002)$.

\section{ALOCAÇÃO DE ENERGIA EM PEIXES}

A alocação de energia tem início com a ingestão do alimento e termina com a deposição de diversas substâncias no corpo do organismo, as quais serão refletidas no crescimento, somático ou reprodutivo. Diversas variáveis bióticas e abióticas atuam sobre o processo final, alterando-o direta ou indiretamente (Pedersen \& Hislop 2001, Dourado \& BeneditoCecíllio 2005). A atuação de algumas variáveis abióticas se distingue em relação aos aspectos regionais, como variação da temperatura, fotoperíodo e sazonalidade (Wootton 1990, Lowe-McConnel 1999). Por exemplo, devido a pouca variação na temperatura da água, espécies de clima tropical canalizam sua energia em um crescimento mais rápido e eficiente (Pandian \& Vivekanandan 1985).

A variação sazonal na densidade de energia em peixes está geralmente associada com os ciclos reprodutivos e alimentares (Hislop et al. 1991, Encina \& Granado-Lorencio 1997). No entanto, devido à dificuldade de se trabalhar com densidade energética em peixes, poucos trabalhos demonstram esta relação (e.g., Benedito-Cecilio \& Morimoto 2002, Vismara et al. 2004, Santos et al. 2006, Monteiro et al. 2007).

Segundo Pedersen \& Hislop (2001), o tamanho do peixe não apresenta relação positiva com a quantidade de energia, no entanto, o peso seco apresenta esta relação, sendo uma medida energética mais adequada. Porém, o peso seco pode apresentar limitações, visto que, segundo Doria \& Andrian (1997) o conteúdo calórico do tecido reprodutivo pode variar durante o desenvolvimento do peixe. Considerando apenas o peso seco, as diferenças entre os tipos de tecido e variações na energia alocada durante o estádio de desenvolvimento são ignoradas. Por isso, parâmetros somáticos como fator de condição e índice gônadosomático, que demonstram forte relação com o conteúdo energético são mais indicados em estudos sobre energia em peixes (e.g Doria \& Andrian 1997, Encina \& Granado-Lorencio 1997).

O estoque de energia também pode variar de acordo com o comportamento do peixe. No caso de migradores, o estoque de energia está muito associado à dieta pois eles estocam energia para grandes períodos migratórios (Saldaña \& Venables 1983), tendo no final da migração um estoque energético muito baixo. No caso de migradores, há um investimento em estoque energético antes da migração, sendo este investimento estocado principalmente em gordura que, posteriormente, será convertida em tecido reprodutivo. É importante ressaltar que a quantidade e a qualidade do alimento disponível irá alterar a entrada de energia no organismo, e conseqüentemente, o processo de alocação energético (Adams et al. 1982, Pandian \&Vivekanandan 1985).

$\mathrm{Na}$ formação de teias de fluxo de energia é importante considerar as variabilidades energéticas associadas a cada compartimento. Essa variabilidade é fundamental ao entendimento do fluxo de energia ao longo da cadeia (Dourado \& Benedito-Cecíllio 2005). Desta forma, as variações de alocação de energia em peixes citadas anteriormente serão extremamente importantes para o entendimento da dinâmica energética do sistema. Em relação às unidades de energia para a formação da teia, a densidade calórica é uma das mais indicadas, devendo ser mensurada para os diferentes integrantes das teias alimentares, visto que variações nas mensurações poderão influenciar significativamente os cálculos do modelo de consumo, produção e eficiência de conversão (Winberg 1971, Economidis et al. 1981).

Os estudos de bioenergética tiveram início no final da década de 40 (Jobling 1994), sendo a maioria dos trabalhos voltada para os peixes (e.g. Mann 1965, Dorian \& Adrian 1997, Arrhenius 1998, Andersen 1998, Pedersen 2000, Pedersen \& Hislop 2001, Benedito-Cecílio \& Morimoto 2002). No Brasil, são poucos os trabalhos existentes com energética de peixes, sendo os primeiros relativos a espécies marinhas (Soares 1990, Ngan 1993). Para ambientes de água doce o número de trabalhos é maior, sendo a maioria na bacia do Paraná (Doria \& Adrian 1997, Penczak et al. 1999, Benedito-Cecilio \& Morimoto 2002 Vismara et al. 2004, Santos et al. 2006, Monteiro et al. 2007). 


\section{ALOCAÇÃO DE ENERGIA POR INVERTE- BRADOS AQUÁTICOS}

Para os invertebrados aquáticos, as maiores variações no conteúdo calórico estão relacionadas com sazonalidade (variação de temperatura) e com a disponibilidade de alimento do que entre as classes dos organismos, como por exemplo, moluscos, insetos e crustáceos (Griffiths 1977, Vannote 1978, Grafius \& Anderson 1979). Um outro aspecto importante a ser considerado é o ciclo de vida que envolve diferentes estádios de desenvolvimento: larva, ninfas, pupas e adultos (Borror \& Delong 2005). O estoque energético para estes estádios pode apresentar diferenciação, visto que há uma maior alocação de energia antecedendo os estádios como os de pupa, onde o organismo não se alimenta. Devido a estas variações, as relações entre tamanho corpóreo e densidade energética não apresentam o mesmo padrão para adultos e larvas (Griffiths 1977).

Para insetos como Ephemeroptera, Trichoptera e Diptera foi observado que ocorre aumento do metabolismo e investimento em crescimento em altas temperaturas, e com a diminuição da temperatura ocorre uma redução no metabolismo, de forma que os indivíduos maiores (com menor taxa de metabolismo) são positivamente selecionados (Vannote 1978, Grafius \& Anderson 1979). Estas variações direcionam o investimento energético dos organismos de acordo com as condições ambientais (Vannote 1978, Grafius \& Anderson 1979).

Assim como para os peixes, as variações na alocação de energia dos invertebrados aquáticos estão relacionadas com aspectos sazonais, comportamentais e com o ciclo de vida dos organismos. Estas variações energéticas são refletidas no compartimento da teia composto por estes organismos, sendo importante considerar os aspectos anteriormente citados (Winberg 1971). Desta forma, em trabalhos com fluxo de energia o ideal é que os dados calorimétricos sejam extraídos do ambiente natural. Tabelas de conversão e relações com biomassa (peso) podem ser utilizadas com cautela, visto que o conteúdo calórico pode variar com o estádio de vida, sexo, idade, estação do ano e dieta dos organismos (Golley 1961, Cummins \& Wuycheck 1971, Griffiths 1977).

\section{TEIAS TRÓFICAS}

Em ambientes lóticos encontramos dois tipos de teias descritas (i) de conectividade também chamada de topológicas ou binária que considera apenas a presença e ausência das ligações tróficas (ii) quantitativas, ou de fluxo de energia que consideram a importância relativa dos componentes consumidos pelas espécies (Townsend et al 1998). Para a construção de teias quantitativas, são necessárias informações sobre taxas de produção e composição da dieta dos organismos envolvidos (Christen \& Pauly 1992, Teng \& McCann 2004). Pela sua simplicidade, as topológicas são consideradas insuficientes para descrever a dinâmica da teia, sendo as de fluxo de energia mais indicada para verificar questões sobre a estabilidade e dinâmica (Teng \& McCann 2004).

As teias quantitativas de fluxo de energia trabalham com distintas unidades de biomassa como: peso úmido, peso seco, volume e densidade calórica (Christensen et al. 2005). A partir da quantificação destes dados é possível se trabalhar com sistemas balanceados com equações de balanceamento de massa (Santana-Porto \& Adrian 2006).

A metodologia convencional para analisar teias quantitativas é baseada em resultados quantitativos da dieta, que podem apresentar problemas devido à taxa de digestão das presas, e dificuldades na identificação de itens amorfos como algas e detritos. Além disso, dados de dieta representam fotografias instantâneas da dieta do organismo. A composição da dieta raramente considera a assimilação das presas, menosprezando presas raras (Elton 1927). Mesmo análises intensivas da dieta podem ser inadequadas para determinar a energia e o fluxo através das teias porque a composição da dieta raramente considera a assimilação das presas, podendo menosprezar presas raras (Schindler \& Lubetkin 2004). Por isso, a técnica dos isótopos estáveis é indicada, para descrever o fluxo de energia no sistema.

No entanto, os trabalhos com teias descritivas foram imprescindíveis para o desenvolvimento da teoria de teias tróficas (Pimm 1982). Essa teoria começou a ser aprimorada com Paine (1966) e May (1972). Segundo Winemiller (1990), apesar de algumas generalizações propostas por May (1972) terem sido falseadas, elas estimularam o desenvolvimento dessa área da ecologia, com isso, surgiram vários trabalhos posteriores (e.g. DeAngelis et al 1980, Cohen 1977, 1978), Pimm (1980, 1982), Yodzis (1981). Na década de 90, Polis (1991) trabalhou em uma teia com 2.000 espécies, que derrubou várias das 
generalizações propostas anteriormente como o limite de complexidade teia, a onivoria como elemento gerador de instabilidade na teia ou comprimento máximo de cadeias na teia como sendo de cinco níveis tróficos. Nesse período foram descritas diversas teias para ambientes lóticos (e.g. Winemiller 1990, Brett \& Goldman 1997, Townsend et al. 1998, Winemiller \& Jepsen 1998, Aioki \& Mizushima 2001, Finlay 2001, Power \& Dietrich 2002, Aoiki 2003, Motta \& Uieda 2005) muitas corroborando os questionamentos de Polis (1991), principalmente em relação à onivoria. No caso dos riachos, é importante ressaltar que a maioria das teias elucidadas destacam a importância da matéria alóctone para o sistema (Polis \& Hurd 1996, Nakano et al. 1999, Nakano \& Murakami 2001, Kawaguchi \& Nakano 2001, Kawaguchi et al. 2003), inclusive enfatizando a importância da preservação da vegetação marginal (Power \& Dietrich 2002). Além da importância da vegetação marginal, a produção primária também se destaca como reguladora das teias de riachos. Efeitos relacionados a sazonalidade destacando a importância da variação seca e chuvosa foram descritos recentemente por (Power et al. 2008) enfatizando a importância das algas e dependência de um predador especifico para o consumo de determinadas algas.

Além das teias descritivas e de fluxo de energia, muitos experimentos para verificar processos e interações entre os organismos de riachos vêm sendo realizados. Dentre os objetivos desses trabalhos se destacam a observação de cascatas tróficas, identificação de espécies-chave e efeitos indiretos. Na região neotropical, esses experimentos comprovaram efeitos diretos e indiretos da remoção do sedimento (Flecker 1996, Pringle 1996, Power 1997, Pringle \& Hamazaki 1998) ou efeitos de cascata trófica no sistema (Pringle \& Blake 1994, Bentead et al. 1999). Existem trabalhos semelhantes aplicados a riachos de Mata Atlântica (e.g. Silveira \& Moulton 2000, Motta \& Uieda 2002, Moulton et al. 2004, Souza et al. 2007). Alguns resultados como os de Silveira \& Moulton (2000) contrariam a teoria proposta por Pimm (1982), demonstrando cascata trófica decorrendo da exclusão de um onívoro.

A discussão sobre teias tróficas ainda é atual, principalmente para a região neotropical, onde não é grande o número de trabalhos e alguns dos trabalhos realizados têm falseado as generalizações anteriores (e.g. Winemiller 1990, Silveira \& Moulton
2000, Motta \& Uieda 2005). Como já apresentado anteriormente, as técnicas de isótopos para descrição de fluxo de energia $\left(\delta^{13} \mathrm{C}\right)$ ou verificação de níveis tróficos $\left(\delta^{15} \mathrm{~N}\right)$ tem trazido inovações principalmente em relação ao fluxo do carbono, redirecionando os trabalhos recentes da área.

\section{CONSIDREÇÃO FINAIS}

A modelagem bioenergética tem se tornado uma poderosa ferramenta em estudos de Ecologia Energética, apresentando abordagens que podem ser aplicadas a nível populacional ou ecossistêmico.

Em nível populacional os trabalhos de alocação de energia em peixes podem ser aplicados na aqüicultura e no manejo das espécies nativas, visto que esses estudos têm dentre seus objetivos verificar: (i) taxas de estocagem (ii) taxas de crescimento (iii) relações entre o consumo e crescimento (iv) taxas de conversão de alimento.

Os isótopos estáveis podem ser aplicados em nível populacional ou ecossistêmico, através da determinação do fluxo de energia pelo $\delta^{13} \mathrm{C}$ ou da posição trófica pelo $\delta^{15} \mathrm{~N}$. Muitos trabalhos vêm sendo desenvolvidos nessa área e novas perguntas acerca da origem do carbono incorporado em teias tróficas de rios estão surgindo. Novos estudos de caso são necessários para verificar estes padrões, no entanto, é importante ressaltar que apesar de ser discutida que a maior fonte de energia seja proveniente das microalgas, a vegetação ripária é imprescindível para o funcionamento do sistema.

Desta forma, a intensificação de estudos sobre fluxo de energia e alocação de energia por espécies aquáticas no Brasil é imprescindível, seja para o manejo de espécies de interesse econômico, seja para a conservação de sistemas aquáticos.

AGRADECIMENTOS: Aos revisores anônimos pelas críticas ao manuscrito e às professoras Miriam Pilz Albrecht e Ana Cristina Petry pelas sugestões e participação da banca de qualificação da presente revisão e Piatã Marques pela revisão de língua inglesa do abstract. Agradecemos também ao $\mathrm{CNPq}$ pelas bolsas individuais de C.F.R. (CNPq 140928/2005-7; CAPES-PDEE 012008-01), E.P.C (CNPq 470587/2004-0) e R.M. (CNPq 311976/2004-0).

\section{REFERÊNCIAS}

ADAMS, S.M.; MCLEAN, R.B. \& PARROTTA, J.A. 1982.

Energy Partitioning in Largemouth Bass under Conditions of 
Seasonally Fluctuating Prey Availability. Transactions of the American Fisheries Society, 111(5): 549-558.

AIOKI, I. 2003. Diversity-productivity-stability relationship in freshwater ecosystems: Whole-systemic view of all trophic levels. Ecological Research, 18: 397-404.

ANDERSEN, N.G. 1998. The effect of meal size on gastric evacuation in whiting. Journal of Fish Biology, 52(4): 743-755.

ANGELINI, R. 2002. Bases para a aplicação da teoria da informação em ecossistemas, com ênfase na ascendência. Acta Scientiarum, 24(2): 275-283.

ANGELINI, R. \& AGOSTINHO, A.A. 2005. Food web model of the Upper Parana River Floodplain: description and aggregation effects. Ecological Modelling, 181(2-3): 109-121.

ARAUJO-LIMA, C.A.R.M.; FORSBERG, B.; VICTORIA, R. \& MARTINELLI, L. 1986. Energy Sources for Detritivorous Fishes in the Amazon. Science, 234(4781): 1256-1258.

ARRHENIUS, F. 1998. Variable length of daily feeding period in bioenergetics modelling: a test with 0-group Baltic herring. Journal of Fish Biology, 52(4): 855-860.

BAPTISTA, J.P. 2006. Os princípios fundamentais ao longo da história da Física. Revista Brasileira de Ensino de Física, 28(4): 541-553.

BENEDITO-CECILIO, E. \& ARAUJO-LIMA, C. 2002. Variation in the carbon isotope composition of Semaprochilodus insignis, a detritivorous fish associated with oligotrophic and eutrophic Amazonian rivers. Journal of Fish Biology, 60(6): 1603-1607.

BENEDITO-CECILIO, E.; ARAUJO-LIMA, C.; FORSBERG, B.R.;BITTENCOURT, M.M. \& MARTINELLI, L.C. 2000. Carbon sources of Amazonian fisheries. Fisheries Management and Ecology, 7(4): 305-314.

BENEDITOCECÍLIO, E. ; DOURADO, E. C. S. ; LOPES, C. A. ; FARIA, A. C. E. A. 2004. Trophic structure of fish assemblage in the floodplain of the upper Parana river: use of stable isotopes. Pp. 145-149. In Agostinho, A.A. Rodrigues, L. Gomes, L.C. Thomaz, S.M. \& , L.E. Miranda, (eds), The upper Paraná river floodplain long term ecological research. Maringá, EDUEM, 210p.

BENEDITO-CECILIO, E. \& MORIMOTO, M. 2002. Effect of preservatives on caloric density in the muscles of Hoplias aff. malabaricus (Bloch, 1794)(Osteichthyes, Erythrinidae). Acta Scientiarum, 24: 112-114.

BENSTEAD, J.P.; MARCH, J.G.; PRINGLE, C.M. \& SCATENA, F.N. 1999. Effects of a low-head dam and water abstraction on migratory tropical stream biota. Ecological Applications, 9(2): 656-668.
BORROR \& DELONG. 2005. Introduction to the Study of Insect Thomson Brooks/Cole. Belmont. 863p.

BRETT, M.T. \& GOLDMAN, C.R. 1997. Consumer versus resource control in freshwater pelagic food webs. Science, 275(5298): 384-386.

BRITO, E.F.; MOULTON, T.P.; DE SOUZA, M.L. \& BUNN, S.E. 2006. Stable isotope analysis indicates microalgae as the predominant food source of fauna in a coastal forest stream, south-east Brazil. Austral Ecology, 31(5): 623-633.

CHECKLEY, D.M. \& ENTZEROTH, L.C. 1985. Elemental and Isotopic Fractionation of Carbon and Nitrogen by Marine, Planktonic Copepods and Implications to the Marine Nitrogen-Cycle. Journal of Plankton Research, 7(4): 553-568.

CHRISTENSEN, V. 1995. Ecosystem Maturity - Towards Quantification. Ecological Modelling, 77(1): 3-32.

CHRISTENSEN, V. \& PAULY, D. 1992. Ecopath-Ii - a Software for Balancing Steady-State Ecosystem Models and Calculating Network Characteristics. Ecological Modelling, 61(3-4): 169-185.

CHRISTENSEN, V.; WALTERS, C.J. \& PAULY, D. (2005). Ecopath with Ecosim: a User's Guide. Vancouver. University of British Columbia. 154p.

COHEN, J.E. 1977. Food Webs and Dimensionality of Trophic Niche Space. Proceedings of the National Academy of Sciences of the United States of America, 74(10): 4533-4536.

CUMMINS, K.W. 1974. Structure and Function of Stream Ecosystems. Bioscience, 24(11): 631-641.

CUMMINS, K.W. \& WUYCHECK, J.C. 1971. Caloric equivalents for investigations in the ecological energetic. Mitt. Int. Verein. Limnol., 18: 1-158.

DEANGELIS, D.L.; SVOBODA, L.J.; CHRISTENSEN, S.W. \& VAUGHAN, D.S. 1980. Stability and Return Times of LeslieMatrices with Density-Dependent Survival - Applications to Fish Populations. Ecological Modelling, 8: 149-163.

DENIRO, M.J. \& EPSTEIN, S. 1978. Influence of Diet on Distribution of Carbon Isotopes in Animals. Geochimica Et Cosmochimica Acta, 42(5): 495-506.

DENIRO, M.J. \& EPSTEIN, S. 1981. Influence of Diet on the Distribution of Nitrogen Isotopes in Animals. Geochimica Et Cosmochimica Acta, 45(3): 341-351.

DORIA, C.R. \& ADRIAN, I.F. 1997. Variation in energy content of somatic and reproductive tissues related to the reproductive cycle and feeding of female Pimelodus maculatus Lacépède, 1803 (Siluriformes, Pimelodidae) and Schizodon borelli Boulenger, 1985 (Characiformes, Anastomidae). Revista Unimar, 19(2): 421-437.

DOURADO, E.C.S. \& BENEDITO-CECILIO, E. 2005. Ecologia 
energética de peixes: influência de fatores abióticos e bióticos. EDUEM, Maringá. 53p.

ECONOMIDS, P.S. 1981. Caloric contents in some freshwater and marine fishes from Greece. Cybim, 5(4): 97-100.

ENCINA, L. \& GRANADO-LORENCIO, C. 1997. Seasonal variations in condition and energy content of somatic and reproductive tissues of Chondrostoma polylepis willkommi. Folia Zoologica, 46: 123-132.

FINLAY, J.C. 2001. Stable-carbon-isotope ratios of river biota: Implications for energy flow in lotic food webs. Ecology, 82(4): 1052-1064.

FISHER, S.G. \& LIKENS, G.E. 1973. Energy Flow in Bear Brook, New Hampshire - Integrative Approach to Stream Ecosystem Metabolism. Ecological Monographs, 43(4): 421-439.

FLECKER, A.S. 1996. Ecosystem engineering by a dominant detritivore in a diverse tropical stream. Ecology, 77(6): 18451854.

FORSBERG, B.R.; ARAUJOLIMA, C.; MARTINELLI, L.A.; VICTORIA, R.L. \& BONASSI, J.A. 1993. Autotrophic Carbon-Sources for Fish of the Central Amazon. Ecology, 74(3): 643-652.

FRY, B. \& ARNOLD, C. 1982. Rapid C-13/C-12 Turnover During Growth of Brown Shrimp (Penaeus-Aztecus). Oecologia, 54(2): 200-204.

FRY, B.; JOERN, A. \& PARKER, P.L. 1978. Grasshopper Food Web Analysis - Use of Carbon Isotope Ratios to Examine Feeding Relationships among Terrestrial Herbivores. Ecology, 59(3): 498-506.

GAEBLER, O.H.; VITTI, T.G. \& VUKMIROV.R. 1966. Isotope Effects in Metabolism of $14 \mathrm{n}$ and $15 \mathrm{n}$ from Unlabeled Dietary Proteins. Canadian Journal of Biochemistry, 44(9): 1249-1251.

GOLLEY, F.B. 1961. Energy Values of Ecological Materials. Ecology, 42(3): 581-590.

GRAFIUS, E. \& ANDERSON, N.H. 1979. Population Dynamics, Bioenergetics, and Role of Lepidostoma Quercina Ross (Trichoptera: Lepidostomatidae) in an Oregon Woodland Stream. Ecology, 60(2): 433-441.

GRIFFITHS, D. 1997. Caloric variation in crustacea and other animals. Journal of Animal Ecology, 46: 593-605.

HAINES, E.B. 1976. Relation between Stable Carbon Isotope Composition of Fiddler Crabs, Plants, and Soils in a SaltMarsh. Limnology and Oceanography, 21(6): 880-883.

HAINES, E.B. \& MONTAGUE, C.L. 1979. Food Sources of Estuarine Invertebrates Analyzed Using C-13-C-12 Ratios. Ecology, 60(1): 48-56.

HAMILTON, S.K.; LEWIS, W.M. \& SIPPEL, S.J. 1992. Energy-Sources for Aquatic Animals in the Orinoco River
Floodplain - Evidence from Stable Isotopes. Oecologia, 89(3): 324-330

HISLOP, J.R.G.; HARRIS, M.P. \& SMITH, J.G.M. 1991. Variation in the Calorific Value and Total Energy Content of the Lesser Sandeel (Ammodytes-Marinus) and Other Fish Preyed on by Seabirds. Journal of Zoology, 224: 501-517.

JOBLING, M. 1994. Fish Bioenergetics. Chapman \& Hall, London. 309p.

KAWAGUCHI, Y. \& NAKANO, S. 2001. Contribution of terrestrial invertebrates to the annual resource budget for salmonids in forest and grassland reaches of a headwater stream. Freshwater Biology, 46(3): 303-316.

KAWAGUCHI, Y.; TANIGUCHI, Y. \& NAKANO, S. 2003. Terrestrial invertebrate inputs determine the local abundance of stream fishes in a forested stream. Ecology, 84(3): 701-708.

KINGSLAND, S.E. 1991. Defining Ecology as a Science. Pp. 1-13. In: L.A. Real \& J.H. Brown (eds) Foundations of Ecology. The University of Chicago Press Chicago.

KLINE, T. C., JR., AND D. PAULY. 1998. Cross-validation of trophic level estimates from a mass balance model of Prince William Sound using $15 \mathrm{Nf} 14 \mathrm{Nd}$ ata. In Proceedingosf the International Symposiumo n Fishery StockA ssessmentM odelse, Alaska Sea Grant College Program Report, Alaska 98-01.

LEITE， R.G.; ARAUJO-LIMA， C.; VICTORIA， R.L. \& MARTINELLI, L.A. 2002. Stable isotope analysis of energy sources for larvae of eight fish species from the Amazon floodplain. Ecology of Freshwater Fish, 11(1): 56-63.

LINDEMAN, R.L. 1942. The trophic dynamic aspect of ecology. Ecology, 23: 399-418.

LOPES, C.A.; BENEDITO-CECILIO, E. \& MARTINELLI, L.A. 2007. Variability in the carbon isotope signature of Prochilodus lineatus (Prochilodontidae, Characiformes) a bottom-feeding fish of the Neotropical region. Journal of Fish Biology, 70(6): 1649-1659.

LOWE-MCCONNELL, R.H. 1999. Estudos Ecológicos de Comunidades de Peixes Tropicais. EDUSP. São Paulo. 534p. MANETTA, G.I. \& BENEDITO-CECILIO, E. 2003. Aplicação da técnica de isótopos estáveis na estimativa da taxa de turnover em estudos ecológicos: uma síntese. Acta Scientiarum, 25(1): 121-129.

MANETTA, G.I.; BENEDITO-CECILIO, E. \& MARTINELLI, M. 2003. Carbon sources and trophic position of the main species of fishes of Baía River, Paraná River floodplain, Brazil. Brazilian Journal of Biology, 63(2): 283-290.

MANN, K.H. 1965. Energy Transformations by a Population of Fish in the River Thames. Journal of Animal Ecology, 34(2): 253-275. 
MANTEL, S.M.K.; SALAS, M. \& DUDGEON, D. 2004. Foodweb structure in a tropical Asian forest stream. Journal of the North American Benthological Society, 23(4): 728-755.

MARCH, J.G. \& PRINGLE, C.M. 2003. Food web structure and basal resource utilization along a tropical island stream continuum, Puerto Rico. Biotropica, 35(1): 84-93.

MEYER, E.I. \& POEPPERL, R. 2004. Assessing food-web structure, matter fluxes, and system attributes of a Central European mountain stream by performing mass-balanced network analysis. Canadian Journal of Fisheries and Aquatic Sciences, 61(9): 1565-1581.

MICHENER, R. \& SCHELL, D. 1994. Stable isotope ratios as tracers in marine aquatic food webs. Stable Isotopes in Ecology and Environmental Science. Blackwell Scientific Publications.

MINAGAWA, M. \& WADA, E. 1984. Stepwise Enrichment of N-15 Along Food-Chains - Further Evidence and the Relation between Delta-N-15 and Animal Age. Geochimica Et Cosmochimica Acta, 48(5): 1135-1140.

MINSHALL, G.W. 1967. Role of Allochthonous Detritus in Trophic Structure of a Woodland Springbrook Community. Ecology, 48(1): 139-\&.

MINSHALL, G.W. 1988. Stream Ecosystem Theory - a Global Perspective. Journal of the North American Benthological Society, 7(4): 263-288.

MONTEIRO, V.; BENEDITO, E. \& DOMINGUES, W.M. 2007. Efeito da estratégia de vida sobre as variações no conteúdo de energia de duas espécies de peixes (Brycon hilarii e Hypophtalmus edentatus), durante o ciclo reprodutivo. Acta Scientiarum, 29: 151-159.

MOSISCH, T.D.; BUNN, S.E. \& DAVIES, P.M. 2001. The relative importance of shading and nutrients on algal production in subtropical streams. Freshwater Biology, 46(9): 1269-1278.

MOTTA, R.L. \& UIEDA, V.S. 2005. Food web structure in a tropical stream ecosystem. Austral Ecology, 30(1): 58-73.

MOULTON, T.P. 2006. Why the world is green, the waters are blue and foodwebs in small streams in the atlantic rainforest are predominantly driven by microalgae? Oecologia Brasiliensis, 10(1): 78-89.

MOULTON, T.P.; SOUZA, M.L.; SILVEIRA， R.M.L. \& KRSULOVIC, F.A.M. 2004. Effects of ephemeropterans and shrimps on periphyton and sediments in a coastal stream (Atlantic forest, Rio de Janeiro, Brazil). Journal of the North American Benthological Society, 23(4): 868-881.

NAKANO, S.;H, M. \& KUHARA, N. 1999. Terrestrial-Aquatic Linkages: Riparian Arthropod Inputs Alter Trophic Cascades in a Stream Food Web. Ecology, 80(7): 2435-2441.

NAKANO, S. \& MURAKAMI, M. 2001. Reciprocal subsidies:
Dynamic interdependence between terrestrial and aquatic food webs. Proceedings of the National Academy of Sciences, 98(1): 166-170.

NGAN, P.V. 1993. Estudos bioenergéticos de animais marinhos costeiros. I Paralonchurus brasiliensis (Perciformes, Sciaenidae). Boletim do Instituto Oceanográfico, 10: 199-215.

ODUM, E.P. 2001. Fundamentos de Ecologia. Fundação Caloustre Gulbenkian. Lisboa. 927p.

ODUM, E.P. \& SMALLEY, A.E. 1959. Comparison of Population Energy Flow of a Herbivorous and a Deposit-Feeding Invertebrate in a Salt Marsh Ecosystem. Proceedings of the National Academy of Sciences, 45(4): 617-622.

ODUM, H.T. 1956. Primary Production in Flowing Waters. Limnology and Oceanography, 1(2): 102-117.

ODUM, H.T. 1957. Primary Production Measurements in Eleven Florida Springs and a Marine Turtle-Grass Community. Limnology and Oceanography, 2(2): 85-97.

ODUM, H.T. \& PINKERTON, R.C. 1955. Times Speed Regulator - the Optimum Efficiency for Maximum Power Output in Physical and Biological Systems. American Scientist, 43(2): 331-343.

PAINE, R.T. 1966. Food Web Complexity and Species Diversity. American Naturalist, 100(910): 65-79.

PANDIAN, T.J. \& VIVEKANANDAN, E. 1985. Energetics of feeding and digestion. Pp. 99-124. P. Tyler and P. Calow Fish Energetics: new perspectives. Croom Helm Sydney.

PATTEN, B.C. 1959. An Introduction to the Cybernetics of the Ecosystem - the Trophic-Dynamic Aspect. Ecology, 40(2): 221-231.

PATTEN, B.C. 1961. Competitive Exclusion - Exclusion Principle Is Recast in Context of a Generalized Scheme for Interspecific Interactions. Science, 134(349): 1599-1600.

PEDERSEN, J. 2000. Food consumption and daily feeding periodicity: comparison between pelagic and demersal whiting in the North Sea. Journal of Fish Biology, 57(2): 402-416.

PEDERSEN, J. \& HISLOP, J.R.G. 2001. Seasonal variations in the energy density of fishes in the North Sea. Journal of Fish Biology, 59(2): 380-389.

PENCZAK, T. 1999. Fish production and food consumption in the Warta River (Poland): continued post-impoundment study (1990-1994). Hydrobiologia, 416: 107-123.

PEREIRA, A.L.; BENEDITO-CECILIO, E. \& SAKURAGUI, C.M. 2007. Spatial variation in the stable isotopes of $13 \mathrm{C}$ and $15 \mathrm{~N}$ and trophic position of Leporinus friderici (Characiformes, Anostomidae) in Corumbá Reservoir, Brazil. Anais da Academia Brasileira de Ciências, 79: 41-49. 
PETERSON, B.J. \& FRY, B. 1987. Stable Isotopes in Ecosystem Studies. Annual Review of Ecology and Systematics, 18: 293-320.

PHILLIPSON, J. 1969. Ecologia Energética. Editora da Universidade de São Paulo. São Paulo. 93p.

PIMM, S.L. 1980. Properties of Food Webs. Ecology, 61(2): 219-225.

PIMM, S.L. 1982. Food Webs Chapman and Hall. London. 219p. POLIS, G.A. 1991. Complex Trophic Interactions in Deserts - an Empirical Critique of Food-Web Theory. American Naturalist, 138(1): 123-155.

POLIS, G.A. \& HURD, S.D. 1996. Linking marine and terrestrial food webs: Allochthonous input from the ocean supports high secondary productivity on small islands and coastal land communities. American Naturalist, 147(3): 396-423.

POLOVINA, J.J. 1984. Model of a Coral-Reef Ecosystem .1. the Ecopath Model and Its Application to French Frigate Shoals. Coral Reefs, 3(1): 1-11.

Pauly, D.;V. Christensen \& C. Walters. 2000. Ecopath, Ecosim, and Ecospace as tools for evaluating ecosystem impact of fisheries. Ices Journal of Marine Science, 57(3): 697-706.

POWER, M.E. 1997. Estimating impacts of a dominant detritivore in a neotropical stream. Trends in Ecology \& Evolution, 12(2): 47-49.

POWER, M.E. \& DIETRICH, W.E. 2002. Food webs in river networks. Ecological Research, 17(4): 451-471.

POWER, M. E.; PARKER, M. S.; DIETRICH, W. E. 2008. Seasonal reassembly of a river food web: Floods, droughts and impacts of fish. Ecological Monographs, 78(2): 263-282.

PRINGLE, C.M. 1996. Atyid shrimps (Decapoda: Atyidae) influence the spatial heterogeneity of algal communities over different scales in tropical montane streams, Puerto Rico. Freshwater Biology, 35(1): 125-140.

PRINGLE, C.M. \& BLAKE, G.A. 1994. Quantitative Effects of Atyid Shrimp (Decapoda, Atyidae) on the Depositional Environment in a Tropical Stream - Use of Electricity for Experimental Exclusion. Canadian Journal of Fisheries and Aquatic Sciences, 51(6): 1443-1450.

PRINGLE, C.M. \& HAMAZAKI, T. 1998. The role of omnivory in a neotropical stream: Separating diurnal and nocturnal effects. Ecology, 79(1): 269-280.

RAU, G.H. 1980. Carbon-13-Carbon-12 Variation in Subalpine Lake Aquatic Insects - Food Source Implications. Canadian Journal of Fisheries and Aquatic Sciences, 37(4): 742-746.

RAU, G.H. 1981. Low N-15-N-14 in Hydrothermal Vent Animals - Ecological Implications. Nature, 289(5797): 484-485.

RAU, G.H. \& ANDERSON, N.H. 1981. Use of C-13-C-12 to Trace Dissolved and Particulate Organic-Matter Utilization by Populations of an Aquatic Invertebrate. Oecologia, 48(1): 19-21.

REZENDE, C.F. \& MAZZONI, R. 2005. Seasonal variation in the input of allochthonous matter in an Atlantic Rain Forest stream, Ilha Grande-RJ. Acta Limnologica Brasiliensia, 17(2): 167-175.

ROSENFELD, J.S. \& ROFF, J.C. 1992. Examination of the Carbon Base in Southern Ontario Streams Using Stable Isotopes. Journal of the North American Benthological Society, 11(1): 1-10.

ROUNICK, J.S. \& WINTERBOURN, M.J. 1986. Stable Carbon Isotopes and Carbon Flow in Ecosystems. Bioscience, 36(3): 171-177.

ROUNICK, J.S.; WINTERBOURN, M.J. \& LYON, G.L. 1982. Differential Utilization of Allochthonous and Autochthonous Inputs by Aquatic Invertebrates in Some New-Zealand Streams - a Stable Carbon Isotope Study. Oikos, 39(2): 191-198.

SALAS, M. \& DUDGEON, D. 2001. Stable-isotope determination of mayfly (Insecta : Ephemeroptera) food sources in three tropical Asian streams. Archiv Fur Hydrobiologie, 151(1): 17-32.

SALDANA, J. \& VENABLES, B. 1983. Energy Compartmentalization in a Migratory Fish, Prochilodus-Mariae (Prochilodontidae), of the Orinoco River. Copeia, (3): 617-623.

SANTANA-PORTO, E.A. \& ADRIAN, I.F. 2006. Utilização do ECOPATH na construção de teias alimentares em ambientes aquáticos. Boletim da Sociedade Brasileira de Ictiologia, 3-5.

SANTOS, M.H.D.; BENEDITO-CECILIO, E. \& DOMINGUES, W.M. 2006. Efeito da maturação gonadal sobre a energia dos músculos de duas espécies de piranhas do reservatório do rio Manso, MT. Acta Scientiarum, 28: 227-236.

SCOTT, D. 1965. The Determination and Use of Thermodynamic Data in Ecology. Ecology, 46(5): 673-680.

SHIEH, J.H. \& FAN, L.T. 1982. Thermodynamic Analysis of a Biomass Pyrolysis Process. Abstracts of Papers of the American Chemical Society, 184(SEP): 63-CELL.

SILVEIRA, R. \& MOULTON, T.P. 2000. Modelling the food web of a stream in Atlantic forest. Acta Limnologica Brasiliensia, 12: 63-71.

SLOBODKIN, L.B. 1960. Ecological Energy Relationships at the Population Level. American Naturalist, 94(876): 213-236.

SOARES, L.S.H. (1990). Alimentação de peixes demersais ao longo do ciclo diário no litoral de Ubatuba, São Paulo: alimento, atividade alimentar e consumo. Tese de Doutorado. Oceanografia Biológica. USP, São Paulo, Brasil. 
SOUZA, M.; MOULTON, T.; SILVEIRA, R.; KRSULOVIC, F. \& BRITO, E. 2007. Responses of Chironomidae (Diptera; Insecta) to the exclusion of shrimps and Ephemeroptera in a coastal forest stream, Rio de Janeiro, Brazil. Brazilian Journal of Biology, 67(1): 47-51.

STEELE, K.W. \& DANIEL, R.M. 1978. Fractionation of Nitrogen Isotopes by Animals - a Further Complication to Use of Variations in Natural Abundance of N-15 for Tracer Studies. Journal of Agricultural Science, 90(FEB): 7-9.

TEERI, J.A. \& SCHOELLER, D.A. 1979. Delta-C-13 Values of an Herbivore and the Ratio of C-3 to C-4 Plant Carbon in Its Diet. Oecologia, 39(2): 197-200.

TENG, J. \& MCCANN, K.S. 2004. Dynamics of compartmented and reticulate food webs in relation to energetic flows. American Naturalist, 164(1): 85-100.

THORP, J.H. \& DELONG, A.D. 2002. Dominance of autochthonous autotrophic carbon in food webs of heterotrophic rivers. Oikos, 96(3): 543-550.

THORP, J.H. \& DELONG, M.D. 1994. The Riverine Productivity Model - an Heuristic View of Carbon-Sources and OrganicProcessing in Large River Ecosystems. Oikos, 70(2): 305-308.

TIESZEN, L.L.; BOUTTON, T.W.; TESDAHL, K.G .\& SLADE, N.A. 1983. Fractionation and Turnover of Stable Carbon Isotopes in Animal-Tissues - Implications for Delta-C-13 Analysis of Diet. Oecologia, 57(1-2): 32-37.

TOWNSEND, C.R.; THOMPSON, R.M.; MCINTOSH, A.R.; KILROY, C.; EDWARDS, E. \& SCARSBROOK, M.R. 1998. Disturbance, resource supply, and food-web architecture in streams. Ecology Letters, 1(3): 200-209.

VANDER-ZANDEN, M.J. \& RASMUSSEN, J.B. 2001. Variation in $\delta 15 \mathrm{~N}$ and $\delta 13 \mathrm{C}$ trophic fractionation: Implications for aquatic food web studies. Limnology and Oceanography, 46(8): 2061-2066.

VANNOTE, R.L. 1978. Geometric Model Describing a QuasiEquilibrium of Energy-Flow in Populations of Stream Insects. Proceedings of the National Academy of Sciences of the United States of America, 75(1): 381-384.

VANNOTE, R.L.; MINSHALL, G.W.; CUMMINS, K.W.; SEDELL, J.R. \& CUSHING, C.E. 1980. River Continuum Concept. Canadian Journal of Fisheries and Aquatic Sciences, 37(1): 130-137.

VAZ, M.M.; PETRERE, J.R.M.; MARTINELLI, L. \& MOZETO,

A.A. 1999. The dietary regime of detritivourous fish from the River Jacaré, Brazil. Fisheries Management and Ecology, 6: 121-132.

VISMARA, M.R.; BENEDITO-CECILIO, E. \& FARIA, A.C.E.A.D. 2004. Efeito da maturação gonadal sobre o conteúdo calórico e condição geral de peixes da planície de inundação do alto rio Acta Scientiarum, 26(2): 189-199.

WAICHMAN, A.V. 1996. Autotrophic carbon sources for heterotrophic bacterioplankton in a floodplain lake of central Amazon. Hydrobiologia, 341(1): 27-36.

WALLACE, J.B.; EGGERT, S.L.; MEYER, J.L. \& WEBSTER, J.R. 1997. Multiple trophic levels of a forest stream linked to terrestrial litter inputs. Science, 277(5322): 102-104.

WANTZEN, K.M.; MACHADO, F.D.; VOSS, M.;BORISS, H. \& JUNK, W.J. 2002. Seasonal isotopic shifts in fish of the Pantanal wetland, Brazil. Aquatic Sciences, 64(3): 239-251.

WIEGERT, R. 1968. Thermodynamic considerations in animal nutrition. The American Society of Zoologists, 8(1): 71-81.

WILSON, J.G. \& PARKES, A. 1998. Network analysis of the energy flow through the Dublin Bay ecosystem. Biology and Environment-Proceedings of the Royal Irish Academy, 98B(3): 179-190.

WINBERG, G.G. 1971. Methods for estimation of production aquatic animals. Academic Press, London. 175p.

WINEMILLER, K.O. 1990. Spatial and Temporal Variation in Tropical Fish Trophic Networks. Ecological Monographs, 60(3): 331-367.

WINEMILLER, K.O. \& JEPSEN, D.B. 1998. Effects of seasonality and fish movement on tropical river food webs. Journal of Fish Biology, 53: 267-296.

WINTERBOURN, M.J.; COWIE, B. \& ROUNICK, J.S. 1984. Food Resources and Ingestion Patterns of Insects Along a WestCoast, South-Island, River System . New Zealand Journal of Marine and Freshwater Research, 18(3): 379-388.

WOOTON, R.J. 1990. Ecology of Teleost Fishes. Chapman \& Hall, London. 238p.

YODZIS, P. 1981. The Stability of Real Ecosystems. Nature, 289(5799): 674-676.

Submetido em 22/08/2008. Aceito em 16/09/2008. 